\title{
Using a dynamic model of technological processes to reduce the cost of heavy oil extraction
}

\author{
Dr. lakov S. Korovin ${ }^{1, a}$, Dr. Anatoly I. Kalyaev1,b, Dr. Maxim V. Khisamutdinov ${ }^{3, c}$ \\ ${ }^{1}$ Scientific research institute of multiprocessor computer systems \\ of the Southern Federal University Taganrog, Russia \\ akorovin_yakov@mail.ru, banatoly@kalyaev.net, ‘L-V-P@yandex.ru
}

Keywords: oil and gas industry, heavy oil, the substitution of import, decision support systems, digital oilfield.

\begin{abstract}
The article considers the problem of reducing the cost of heavy oil productions. A new approach, based on the intelligent analysis of successful events' historical data of qualitative EOR application, is proposed. Based on database info analysis, the synthesis of a model, targeted to automated search of wells for EOR application, is worked out. The main data processing tool is a novel technology, based on neural network analysis techniques and evolutionary algorithms implementation. This approach allows selecting EOR in fuzzy, hardly formalized oilfield conditions and reduces the dependence on the human factor.
\end{abstract}

\section{Introduction}

One of the most important trends observed in today's global oil sector is reducing of production at easily accessible and highly productive fields. Operating conditions are complicated fields with the transition to the development of deposits located at greater depths at abnormally high reservoir pressures and temperatures. Increased recovery rates of gas, condensate and oil from tight reservoirs of low permeability is determined using complex methods of stimulation. Considering this, and taking into account the low prices of hydrocarbons, the feasibility of mining the oil is gaining more importance in the world economy.

To maintain current levels and increase the need to examine in more detail the issue of deep drilling new approaches to the production of oil have to be developed, because it requires a major review of all established geological concepts. Future projects for deep drilling will create above all a unique industrial and technological base [1].

The deterioration of the structure of oil reserves over the past decade and a half has led to a decrease in flow rates, complication of the conditions of their operation, which, caused a reducing of turnaround time and service life of mining equipment, increased equipment cost special versions. Switch to low-productivity equipment is accompanied by a rise in unit costs of oil production. The consequence of these processes is a steady decline in the profitability of oil production. Operating costs for the extraction of heavy oil and natural bitumen is 3-4 times higher than the cost of production of light oil, which is associated not only with higher density and viscosity of heavy oils, but with the lack of development of the technology of extraction and processing. Therefore, development of new technologies for the production of heavy and extra-viscous oil is a priority for the development of the entire oil industry.

An analysis of the current state of the oil and gas industry in the light of the problem of reducing the cost of production of heavy oil field development. The analysis methods, models and approaches used in Western Siberia, has shown that the technology used, unfortunately, are, on the one hand, is largely obsolete (attempts to create a "classic" model, based on complex mathematical calculations, based on the works of Russian specialists 60-70 years of the 20th century), and, on the other hand the use of expensive (cost reaches tens of millions of US dollars) software. Among them leaders, as well as in 
other areas of automation of oil production, are the solutions Schlumberger, Halliburton and Weatherford. Among the factors complicating the application of numerical simulation methods on objects of oil and gas companies can be attributed noisy data telemetry, and the actual differences in the design of solutions in the field of drilling and well control modes, other errors, including those determined by the human factor. The totality of unaccounted factors affecting the oil production process, as well as a wide range of difficult to formalize the parameters determining the results of mathematical modeling in the prior art (in particular, products Schlumberger) makes it difficult to build an adequate model for the task.

\section{New approach for reducing of the cost of heavy oil extraction}

In this context, we propose an approach of selecting the optimal measures to increase oil recovery, based on analysis of historical data on production processes that take place in the field, with the application of modern methods of mining data combined. This approach has been successfully tested in a group of authors solve the problem of operative warning of possible failures of equipment high-producing oil wells. The present research is aimed at modification of methods and algorithms, as well as developing new ones with a view to achieving the goal of optimization of the process of the activities designed to enhance oil recovery, thus making the manufacturing process more cost-effective (this is especially true in the current era of low prices for hydrocarbons). Consider the proposed approach in detail.

As practice shows, conducting enhanced oil recovery (EOR) in most cases is in itself an expensive process, and if the final selection event is wrong, the losses can be enormous. Therefore, all information on the carrying out of EOR, applied types of activities, geological and physical data, data on wells and their respective environmental parameters have to be collected in a database for later analysis. This retrospective, which can be represented as an array of data that stores parameters with the corresponding outcome is the data source for the formation of the core system.

Functional diagram of the procedure system settings (core training or formation of the initial profile) are shown in Fig. 1.

All available data of the database containing information on the outcome of the EOR should be used for the analysis. Initially, the array is divided into two parts of equal proportions (an approach has been used successfully in the theory of intelligent information analysis). The first half of the array is exposed to a multi-stage processing. Consider the process in more detail (Fig. 1).

Practice shows that the "raw" data status DB oilfield in the original form are not very suitable for the formation of the profile of the model, which can be used for recognition of new information on the results of learning. In this regard, such data are cleaning procedures and checks for completeness.

Cleaning is the removal of the noise (the "information trash" - data that are knowingly false and contradictory) Check for completeness - is to control the filling in the database with the necessary information. If any of the fields are empty, they are automatically removed from the sample (usually handle these fields is set by experts in the subject area of research) [2]. After that, there is clustering methods k-means and forel [3]. The purpose of the clustering stage is to identify the classes that will be used as reference during the training phase of the model [4]. 


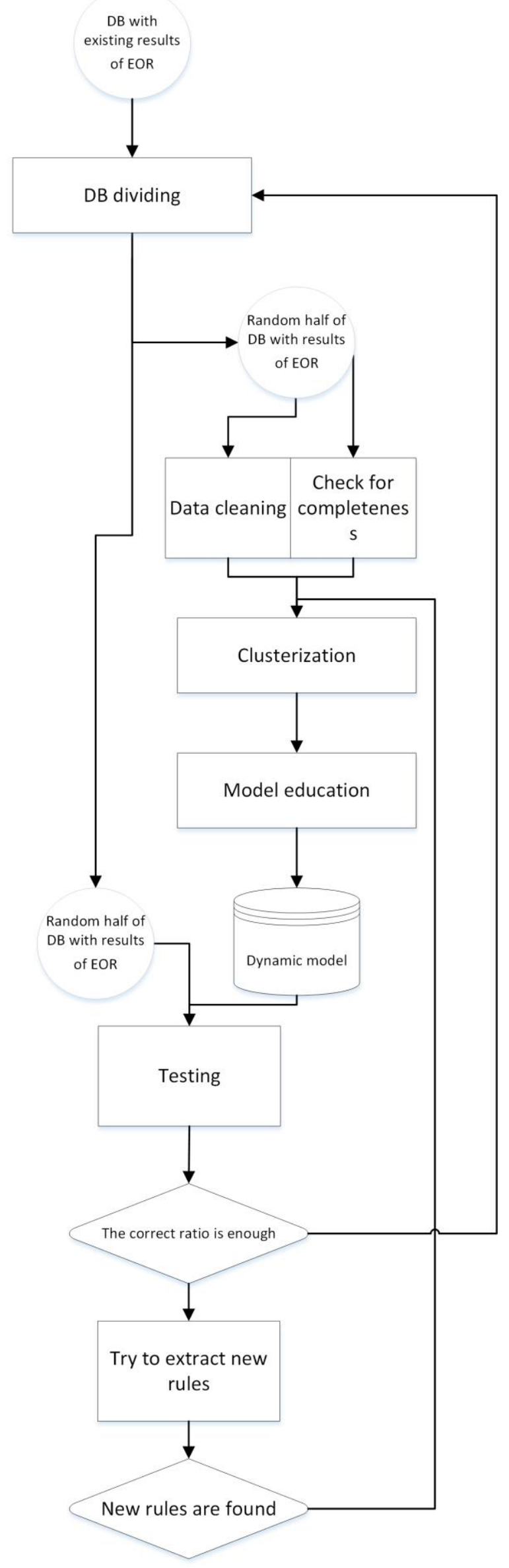

Figure 1. The Functional diagram of the procedure for preparing the core of intelligent selection of optimum EOR 


\section{Conclusions}

Developed method of intelligent processing of data to minimize the human factor error and mathematical models allows adapting to the conditions of the manufacturing process based on an analysis of historical retrospective application of EOR similar within the parameters of the bushes and fixing hidden patterns in complex telemetry databases.

The proposed intelligent approach involves the collection and processing of historical information about the ongoing effects on the formation and long-term effects of the operations in machine-readable form (in the form of relativistic database). The practice of such decisions shows low effectiveness in the situation of a small amount of historical samples, so that a preliminary stage of implementation of intelligent systems is a deep systematization and automation of the management of digital documents both of the studies of rocks, and on fixing the operational performance parameters, as well as physical is the chemical properties of the extracted products.

Further work of authors will focus on the experimental study of the developed methods, approaches and algorithms using real production data.

\section{Acknowledgment}

This paper is published with the financial support of the Russian Science Foundation (RSF) via the grant № 15-19-00196.

\section{References}

[1] P. Danilov. Effect of the oil industry on the environment. Work. Summary on the topic: The impact of the oil industry on the environment // Summary MSTU "STANKIN" Department of Environmental Engineering. Information

[2] Korovin, Y.S., Tkachenko, M.G., Kononov, S.V. (2012) Oilfield Equipment's State Diagnostics on the Basis of Data Mining Technologies, (9), pp. 116-118.(In Russ.) Neftyanoe khozyaystvo = Oil Industry

[3] I.S. Korovin, M.V. Khisamutdinov. Neuronetwork decision support system for oilfield equipment condition online monitoring. Advanced Materials Research (Trans Tech Publications, Switzerland). Volume 902 (2014), Pages 409-415.

[4] Korovin, Y.S., Khisamutdinov, M.V., Tkachenko, M.G. (2013) Forecasting of Oilfield Equipment Work Conditions with the Application of Evolutionary Algorithms and Artificial Neural Networks, (12), pp. 128-133.(In Russ.) Neftyanoe khozyaystvo = Oil Industry 